EPJ Web of Conferences 53, 02003 (2013)

DOI: $10.1051 /$ epjconf/20135302003

(C) Owned by the authors, published by EDP Sciences, 2013

\title{
Some closing marks made at the CERN symposium on future directions in UHECR physics
}

\author{
A.A. Watson \\ School of Physics and Astronomy, University of Leeds, Leeds LS2 9JT, UK
}

\begin{abstract}
Some comments are made about the Symposium held in CERN in February 2012 to discuss future directions for research in ultra-high energy cosmic rays.
\end{abstract}

\section{INTRODUCTION}

This was a highly-enjoyable meeting. Reflecting on it, it is really remarkable that a discussion on the status and future of ultra-high energy cosmic rays (UHECR) should have taken place in this temple of particle physics. It would not have happened 40 years ago in those dark days when cosmic-ray physics was held in extremely low regard by the particle-physics community. And that community had some justification for their views: from cosmic-ray work very high $\mathrm{p}_{t}$ events had been reported (1960s), the free quark had been discovered (1969) and observation of a monopole was claimed (1975). I once overheard a particle physicist, after hearing of one such discovery, give the response 'of course everything has been discovered in cosmic rays': this was not meant kindly. I was once told by a distinguished theoretician 'I never thought cosmic rays were of use for anything'.

Now things are very different. We are here; the Auger Observatory is one of CERN's recognised projects - and has benefited greatly from that status; we have heard talks from CERN physicists who came both to inform and to learn; in some aspects of CMS analysis the hadronic models developed by cosmic-ray people are being used and indeed some of these models fitted better the first LHC data on rapidity than did those developed by particle theorists. Life has changed.

\section{SOME HIGHLIGHTS}

The Symposium has been a great success and the talks were almost always of a very high standard. In particular there were some excellent presentations by astrophysical theorists. However I feel bound to say that I do not believe that UHECR is a field in which theorists really guide the experimental program. At lower energies the nature of the knee, and now the existence of a second knee, have been resolved by high-quality experimentation: now the theorists have their chance to expound and explain but they did not drive the direction of the observations. Similarly the existence of the ankle at a few times $10^{18} \mathrm{eV}$ was discovered experimentally nearly 50 years ago; it was not predicted and it is better experiments, in particular those targeted at determining the mass and the arrival direction distributions, that will solve this riddle. Only at the highest energies has the prediction of the GZK-effect driven experimentation but there was no prediction of the flux at the key energy. Now that we have seen a suppression of the particle flux at roughly the predicted place we need mass and anisotropy measurements to help us learn whether the suppression is the GZK effect or not.

This is an Open Access article distributed under the terms of the Creative Commons Attribution License 2.0, which permits unrestricted use, distribution, and reproduction in any medium, provided the original work is properly cited. 


\section{EPJ Web of Conferences}

Amongst the highlights of the meetings were the reports from Working Groups, made up of members of the Auger, HiRes, Telescope Array and Yakutsk collaborations. The Groups have been established in an effort to resolve the differences between the results reported by the various teams on the energy spectrum, the mass composition, the arrival direction distributions and multi-messenger detections. In addition there was a Working Group report on modelling and high-energy hadronic interactions. That these Working Groups functioned so effectively augurs well for the future.

Of particular note is the report of the Working Group on the mass composition of UHECR presented by Bellido [1]. For some time, differences have been apparent between the inferences made about the primary mass from depth-of-maximum measurements made by HiRes and by the Auger Collaboration. While the Auger group have argued that the mean mass becomes increasingly heavy above $\sim 3 \times 10^{18} \mathrm{eV}$ [2] the HiRes group have claimed over many years that the mass composition is proton-dominated above a similar energy [3]. More recently the Telescope Array (TA) team have reported preliminary results [4] that appear to support the HiRes claims. After scrutinising the analysis procedures of the three groups, the Working Group concluded that there is no evidence to suggest that the apparent discrepancies are due to the different approaches but that there are important differences as to how the conclusions are drawn. In the case of the Auger Observatory, data from all zenith angles are combined directly and compared with model predictions: no differences are found between different zenith angle bands suggesting that the selection criteria are properly understood. In the case of HiRes and the Telescope Array the selection procedures are included as part of the Monte Carlo comparisons with the data. These contrasting approaches make it impossible to compare the separate measurements of $\mathrm{X}_{\max }$ directly. The Yakutsk method of deducing $\mathrm{X}_{\max }$ is to juxtapose the lateral distribution of Cherenkov light with model predictions and extract $\mathrm{X}_{\max }$ indirectly.

To compare the data the Working Group have selected a particular model of hadronic physics, Sibyll, and deduced the mean value of the logarithm of the atomic mass, $\langle\ln \mathrm{A}\rangle$, measurement-bymeasurement. Then a test is made to see whether the data from each observatory fit better the hypothesis of $\langle\ln \mathrm{A}\rangle$ being independent of energy or the hypothesis of a two-component fit with a break in energy at $\log \mathrm{E}(\mathrm{eV})=10^{18.5}$. Comparison between these alternatives has been made using a $\chi^{2}$ test. The Auger data are much better fitted by the two-component model $\left(\chi^{2} / \mathrm{deg}\right.$ of freedom $=133.6 / 10$ (energy independence) and 7.4/9 (two-component fit), while for HiRes (4.4/7 and 1.23/6), TA (9.8/7 and 3.37/6) and Yakutsk (7.7/7 and 4.22/8) the three data sets are consistent with either model. What is clear is that around 4 times more events are needed from the Northern Hemisphere observatories to help resolve this issue. Furthermore data from other observables made with the surface arrays (muon densities in the case of Yakutsk and two independent approaches using the FADC traces from the Auger water-Cherenkov detectors), when treated in a similar manner, indicate that $\langle\ln \mathrm{A}\rangle$ is not constant with energy and that the mean mass becomes heavier as the energy increases. It is important to recognise that quite different systematic uncertainties are associated with the $\mathrm{X}_{\max }$ measurements and with those from the surface array measurements. In my view the Auger two-component model is favoured : unless there is a dramatic change in some features of hadronic interactions at energies beyond the LHC, the mean mass of the highest energy cosmic rays increases as the energy rises.

The Working Group on the energy spectrum was charged with examining the results and methods used to derive the various spectra [5]. They concluded, adopting scaling factors within the systematic uncertainties of the energy scales reported, that the spectra from Auger, HiRes, TA and Yakutsk are consistent in normalisation and shape. To generate spectra that are in agreement they assumed that the differences arise solely from the energy scales adopted. By adjusting these energies by different amounts (Auger $\times 1.102, \mathrm{TA} \times 0.906$, Yakutsk $\times 0.561$, HiResI $\times 0.911$ HiResII $\times 0.903)$ good agreement in the intensities on a plot of $\log$ EJ vs. $\log \mathrm{E}$ is obtained. In my view this is only a first step as attention needs to be given to the sensitivity of the aperture with energy, particularly in the case of HiRes and TA who, when calculating their exposures, have made the assumption of a composition dominated by protons which made well not be securely based $[1,6]$. 
I believe that it is highly desirable for the Working Group on the Energy Spectrum to move next to a comparison of spectra from the same part of the sky. There are useful overlaps with TA and Auger and between TA and Yakutsk. I believe that there is much to be learned by making such comparisons and this would at the same time explore whether or not the fluxes seen in the Northern and Southern hemispheres are different as they might be if, for example, a local source such as Cen A is dominant in the data set recorded at the Auger Observatory.

\section{FEATURES OF HADRONIC INTERACTIONS}

The data on $\mathrm{X}_{\max }$ measured at the Auger Observatory have been used to exploit a method of measuring the cross-section for proton-air collisions first introduced by the Fly's Eye group [7]. The result from Auger at a centre-of-mass energy of $57 \mathrm{TeV}$ was reported [8]. In a clear and provocative discussion of this matter Lipari argued that more information might be extracted from the fluorescence data.

Yushkov, for the Auger Collaboration [9], discussed the observations made in several independent ways that indicate that the muon content in large air-showers is higher than expected from a range of models and mass compositions. The excess is about $40 \%$ in the vertical direction rising to nearly $90 \%$ at larger zenith angles.

The muon observations, coupled with the fact that energy estimates made of primary particles using models are $\sim 30 \%$ higher than those derived from fluorescence calibrations $[5,9]$ by the Auger and TA groups, indicate that although the models of high-energy interactions give a reasonable description of broad features of showers, not all of the details can be explained. This prompts an important new line of investigation with shower observatories in addition to the more traditional astrophysical studies.

\section{THE FUTURE: HADRONIC PHYSICS AND ASTROPHYSICS AT THE HIGHEST ENERGIES}

We are starting to see that detailed measurements of extensive air showers now possible with hybrid instruments such as the Auger Observatory and the Telescope Array hold hope for testing some predictions of hadronic interactions at energies that are unlikely to be reached with terrestrial accelerators. UHECR will always be at the energy frontier although it will never be possible to extract more than the broadest features of hadronic interactions (cross-section, multiplicity and transverse momentum) by studying them. Accordingly discussions are underway to improve the capabilities of the Auger Observatory for observing muons across the whole area of the array. One interesting idea for modifying the water-Cherenkov detectors to have a black top and segmentation was discussed by Letessier-Selvon [10]. It is claimed that with this approach the muon identification will be enhanced in an economical manner. RPCs are also being proposed as a suitable tool. An ambitious scheme to use these devices was described [11] which complements another RPC plan presented earlier this year [12]. It is clear that several lines of $R \& D$ should be explored before deciding which is the most promising.

It was recognised soon after the first measurements of the energy spectrum were made by the Auger Collaboration in 2005 that the aperture was at least one order of magnitude too small. Given that Jim Cronin and I were often asked when pushing the project "why do you want to make it so large?" this has been a huge disappointment and arose because earlier estimates of the UHECR flux had been too high. The challenge therefore is to find an economical method of increasing the aperture by around a factor of 10 or more. One approach is to take a fluorescence detector into space, as proposed by Linsley in the early 1980s, and plans are well-advanced through the JEM-EUSO project to do just that. Several relevant talks were presented at this meeting [13] and it is hoped that the JEM-EUSO instrument will be attached to the Japanese module on the International Space Station in 2016. This extremely ambitious mission has three major goals. Firstly if neutrinos exist with energies above $10^{20} \mathrm{eV}$ they will be detectable with JEM-EUSO: this would have a dramatic impact on astroparticle physics. Secondly it is anticipated that sources of UHECR will be identified. Thirdly the energy spectrum can be pushed to higher energies 
because of the large exposure. Where this instrument is more limited is in the assignment of atomic masses as the accuracy of the determination of $\mathrm{X}_{\max }$ in individual events cannot approach that of hybrid arrays on the ground.

Thus one can envisage a future as follows. On the ground the Pierre Auger Observatory and the Telescope Array will be enhanced, extending their measurement scope to embrace the study of features of hadronic interactions while continuing to enhance their astrophysical data. In space the JEM-EUSO mission will be used to identify neutrinos, extend the cosmic-ray spectrum and find sources of charged cosmic rays. The space observations will need to be followed up by a dedicated ground array of enormous size covering more than $30,000 \mathrm{~km}^{2}$ and of a hybrid nature. The scale of this project can surely only be achieved through a World Observatory perhaps to be realised in 8 to 10 years, but it is not too early to discuss plans, form collaborations and carry out R\&D. I think of this World Observatory as being analogous to the LEP machine and the associated detectors that were built to study the $\mathrm{W}$ and $\mathrm{Z}$ properties in exquisite detail: JEM-EUSO might be thought of being analogous to the SPS. Of course costs must be kept as low as possible for a World Observatory to be supported and an innovative design for a cheaper fluorescence detector was discussed at the meeting by Privitera [14].

The concept of a World Observatory will only become a reality if a team of dedicated people decide to devote a significant part of their careers to making it happen. Dynamic young scientists exist within the several collaborations that have produced the exciting data reported at this meeting. A few must step forward and lead the next stage. JEM-EUSO and a World Observatory must be seen as going hand-inhand to the future: these projects, nor the existing ones on the ground, are not in competition. I believe that the Working Groups are an exciting first step for this adventure but the same sort of cooperation in hardware development is going to be needed if progress is to be made. Tremendous efforts in the last few decades have built on the work of the pioneers of the 1950s and 1960s [15]: I believe that we are now in excellent shape to take the similarly exciting steps.

I have enjoyed useful interactions with members of the mass and spectrum Working Groups but the views expressed on these topics are my own. It is a pleasure to acknowledge the financial support of the Leverhulme Foundation.

\section{References}

[1] E. Baracikowski et al., Report of Working Group on Mass Composition, these Proceedings 2012

[2] J. Abraham et al., (the Pierre Auger Collaboration), Phys. Rev. Lett. 104091101 (2010)

[3] R. Abbasi et al., (the HiRes Collaboration), Phys. Rev. Lett. 104161101 (2010)

[4] C. Jui et al., (the TA Collaboration), Proc APS DPF Meeting, arXiv: 1110.0133

[5] B. Dawson et al., Report of the Working Group on the Energy Spectrum, these Proceedings 2012

[6] A. A. Watson, Reports on Progress in Physics, High-energy cosmic rays and the GreisenZatsepin-Kuz'min effect, 2012 (to be submitted)

[7] R. Ellsworth et al., Phys. Rev. D 263361982 R. Baltrusaitis et al., Phys. Rev. Lett. 521380 (1984)

[8] R. Ulrich for the Pierre Auger Collaboration, Measurement of the proton-air cross-section with the Pierre Auger Observatory, these Proceedings 2012 P Abreu et al., Phys. Rev. Lett. 109062002 (2012)

[9] A. Yushkov, for the Pierre Auger Collaboration, Measurement of the muon shower content at the Pierre Auger Observatory, these Proceedings 2012

[10] A. Letessier-Selvon, UHE particle physics and astrophysics: the need for multi-component EAS measurements and primary particle identification, these Proceedings 2012

[11] M. Ambrosio et al., (the Napoli group), Reconstruction of muon-production depths in ground arrays based on the Time Track Complementary Method, these Proceedings 2012 


\section{UHECR 2012}

[12] L. Lopes, P. Fonte, M. Pimenta, Study of RPCs for autonomous stations in cosmic ray research PoS(RPC2012)043

[13] A. Santangelo, for the JEM-EUSO Collaboration, The JEM-EUSO mission: Context and Status, these Proceedings 2012 T. Ebizusaki, Space-based Observations of Extensive Air Showers, these Proceedings 2012 E. Parizot et al., On the Astrophysical value of larger yet achievable UHECR Detectors, these Proceedings 2012

[14] P. Privitera, A conceptual design for a large ground array of fluorescence detectors, these Proceedings 2012

[15] For a history of the evolution of techniques to study Extensive Air Showers, see K- H. Kampert and A. A. Watson, Extensive Air-Showers and Ultra-high energy cosmic rays: a historical review, Eur. Phys. J. H 37359 (2012), arXiv 1207.4827 